\title{
Plantas utilizadas como cercas vivas en el estado de Veracruz
}

\author{
Sergio Avendaño Reyes ${ }^{1}$ \\ Israel Acosta Rosado ${ }^{1}$
}

\begin{abstract}
RESUMEN
Actualmente la disponibilidad de información sobre plantas empleadas como cercas vivas en el estado de Veracruz es insuficiente. Por tal motivo, el objetivo de este estudio fue el de realizar un inventario para dar a conocer las especies que generalmente son utilizadas para estos fines. El trabajo se desarrolló con base en observaciones de campo, recolecta de material botánico, entrevistas, revisión de ejemplares de herbario y consulta a la base computarizada de datos florísticos de Veracruz. Como resultado se obtuvo un listado de 218 especies de plantas vasculares, el cual es presentado en orden alfabético de familias; se incluyen nombres vulgares, tipos de vegetación y usos. Es importante destacar que el $88 \%$ de las especies registradas presenta diversas categorías de uso adicional; así se obtuvieron 62 especies comestibles, 55 medicinales, 45 ornamentales, 36 de uso como combustible, 27 en construcciones rurales, 22 maderables, 13 como forraje, 6 melíferas y 42 asignadas a otras categorías de uso. Se hace énfasis en la importancia de usar especies nativas dentro de esta práctica agroforestal por contribuir al aprovechamiento adecuado de la flora y a la conservación del germoplasma local.
\end{abstract}

PALABRAS CLAVE:

Cercas vivas, Veracruz, plantas útiles, flora, vegetación.

\begin{abstract}
The available information on species used for live fences in Veracruz is insufficient. Because of this, the objective of this study was to produce an inventory of the species generally used. The study was based on field observations, interviews, collection of botanical material and consulting of herbarium vouchers, as well as the data base on floristic data for Veracruz. A list of 218 species of vascular plants was obtained as a result of this study. This is presented in alphabetical order of families. Common names, vegetation types and uses are also included. It is important to stress that $88 \%$ of the species reported present diverse categories of additional use: 62 species were reported as edible; 55 as medicinal; 45 as ornamental; 36 as fuel; 27 used in rural construction; 22 as timber; 13 as fodder; 6 for honey production and 42 assigned to other categories of use. The use of native species within this agroforestry practice as a contribution to the adequate use of the flora and local germoplasm conservation has been emphasized.
\end{abstract}

KEY WORDS:

Living fences, Veracruz, useful plants, flora, vegetation.

1 Instituto de Ecología, A. C. Departamento de Sistemática Vegetal. Km 2.5 antigua carretera a Coatepec. Apdo Postal 63. 91000 Xalapa, Ver. México.

Manuscrito recibido el 23 de septiembre de 1999. Aceptado el 22 de octubre de 1999 


\section{INTRODUCCION}

La utilización de especies vegetales como cercas vivas constituye una práctica agroforestal que los campesinos han venido manejando con el fin de brindar protección y servir de linderos en espacios dedicados a los cultivos y a la ganadería. En México no se conoce con exactitud desde cuándo se inició esta costumbre dado que en la época anterior a la conquista no hay evidencias palpables que indiquen su uso tradicional. Challenger (1998) menciona que en la época precolombina no se necesitaba protección contra los herbívoros domésticos, pues éstos se desconocían antes de la llegada de los españoles, además de que las tierras eran comunales. Por otra parte, señala que es posible que las cercas vivas sólo se hayan usado en forma incipiente en algunos casos, por ejemplo, como cortinas rompevientos en áreas muy expuestas a la acción de los vientos, principalmente en la época de nortes y ciclones. El mismo autor argumenta también que el uso de cercas vivas en nuestro país, parece ser una práctica posterior a la conquista, derivada de la propagación de especies principalmente útiles o quizá como componente de la construcción de terrazas de cultivo en laderas para que las raíces de las plantas fijaran el suelo; sin embargo, lo que resulta indudable es que la historia de esa técnica es incierta.

En la actualidad el empleo de cercas vivas se encuentra muy extendido particularmente en la áreas rurales. Este sistema agroforestal está constituido por especies que son seleccionadas y plantadas de acuerdo con su durabilidad, con las características ecológicas del sitio y con las posibilidades económicas de los dueños de los terrenos para ser establecidas. Entre las principales ventajas que presentan se encuentran las siguientes: a) constituyen una fuente alternativa de obtención de diversos satisfactores (frutos comestibles, forraje, leña, postes, medicamentos, ornato); b) proporcionan sombra tanto a jornaleros como animales domésticos; c) reducen la fuerza de los vientos y la evaporación; d) ayudan a conservar el suelo y algunas especies favorecen la fijación del nitrógeno; e) evitan la invasión de malezas, f) generan materia orgánica; g) contribuyen a la repoblación y al establecimiento de cercas en otros sitios; h) los costos de plantación a largo plazo resultan más redituables que el empleo de cercas muertas (Romero et al., 1991; Beer, 1994; Otárola, 1995).

Otro aporte importante de las cercas vivas es el papel que desempeñan al funcionar como corredores biológicos de gran relevancia para el paso, refugio y establecimiento de especies faunísticas. Así se encuentran aves, mamíferos (murciélagos, roedores), reptiles e insectos (hemípteros e himenópteros), que a su vez llevan a cabo diferentes interacciones ecológicas al actuar como agentes dispersores de semillas o polinizadores tanto de las propias cercas como de los cultivos involucrados (Challenger, 1998; Maldonado et al., 1998). Además las cercas vivas albergan numerosas especies epífitas como orquídeas, bromelias, cactáceas, piperáceas, helechos, musgos, etc.

El uso de cercas vivas ha sido escasamente estudiado en nuestro país; entre algunos de los trabajos realizados se encuentran los siguientes: Alavez (1983), Chacón (1994), García (1993) y Maldonado et al. (1998), donde se mencionan las especies empleadas para este fin en el estado de Tabasco; el de López (1990) que aborda las especies utilizadas en la zona árida de Chihuahua; los estudios de especies útiles de Chulim y Tun (1998) y de germinación de seis especies de Salinas y Tun (1998), llevados a cabo en la Península de Yucatán. En el estado de Veracruz no se han efectuado inventarios de cercas vivas, solamente han sido consideradas como un apartado dentro de los estudios florísticos municipales realizados por Castillo-Campos (1991), Luna (1997) y Zamora y Castillo- 
Campos (1998), que incluyen 33, 22 y 55 especies respectivamente.

Considerando el alto índice de destrucción de nuestros recursos naturales, propiciado por la apertura de espacios para la ganadería, agricultura, el desarrollo industrial y la tendencia hacia la expansión de áreas urbanas, se hace necesaria la tarea de rescatar e impulsar prácticas como la de cercas vivas. En las comunidades la escasez de especies arbóreas cada vez es mayor, lo que implica que el costo por acarreo de postes de los manchones retirados del sitio donde van a ser colocados afecta seriamente las posibilidades económicas de los propietarios de predios (Zamora y CastilloCampos, 1998); de aquí la importancia de fomentar el uso de especies vegetales como cercas vivas. La disponibilidad de información sobre cuáles especies están siendo utilizadas para este fin, en qué tipos de vegetación se distribuyen y los usos adicionales que de ellas se obtienen, constituyen el punto de partida hacia su conocimiento y aprovechamiento agroforestal adecuado.

\section{OBJETIVO}

Integrar un inventario de las especies vegetales utilizadas como cercas vivas en el estado de Veracruz con el fin de contribuir al conocimiento y fomento de esta práctica agroforestal.

\section{METODO}

El trabajo fue realizado a través de observaciones y entrevistas en el campo, recolecta de material botánico, revisión de ejemplares de herbario y consulta a la base computarizada de datos florísticos de Veracruz (Instituto de Ecología, A.C., 1994). El material recolectado fue determinado por los autores en el Herbario XAL.

\section{RESULTADOS}

Se registró un total de 218 especies de plantas vasculares utilizadas como componentes de cercas vivas, de las cuales un $9.17 \%$ (20) son introducidas y el $88 \%$ presenta diversas categorías de uso adicional. Así se encontraron 62 especies comestibles, 55 medicinales, 45 ornamentales, 36 de uso como combustible (leña o carbón), 27 en construcciones rurales, 22 maderables, 13 como forraje, 6 melíferas y 42 están asignadas a otras categorías de uso (Fig. 1, Anexo 1). Cabe aclarar que algunas de las especies presentan más de un uso.

Por otra parte, la forma biológica más utilizada corresponde a los árboles con 157 especies; le siguen en importancia los arbustos con 49. Con respecto a los tipos de vegetación donde se presentan las especies, se obtuvieron en el siguiente orden decreciente: 115 en selva baja caducifolia, 73 en selva alta perennifolia, 73 en bosque caducifolio, 69 en encinar, 66 en selva mediana subperennifolia, 31 en selva mediana subcaducifolia, 27 en encinarpinar, 25 en dunas costeras, 18 en pinar, 11 en selva baja espinosa, 9 en manglar y 3 en matorral.

Las familias con mayor número de especies destinadas a servir como cercas vivas corresponden a las Leguminosae con 43, Euphorbiaceae, Fagaceae y Solanaceae con 8, y Apocynaceae y Poaceae con 7. Las especies más utilizadas corresponden a las siguientes: Acacia farnesiana, Aechmea bracteata, Agave angustifolia, A. lophanta, Annona cherimola, Bahuinia divaricata, Bromelia pinguin, Brugmansia suaveolens, Bursera simaruba, Caesalpinia cacalaco, $C$. velutina, Calliandra houstoniana, Casuarina cunninghamiana, C. equisetifolia, Cordia alliodora, Cordia dentata, Crescentia alata, C. cujete, Cupressus benthamii, Duranta repens, Enterolobium cyclocarpum, Eriobotrya japonica, Gliricidia sepium, 


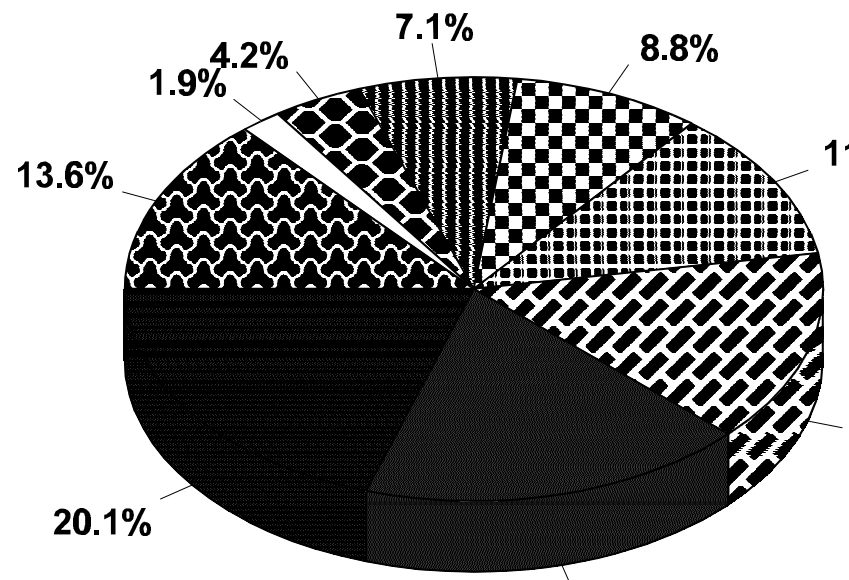

Comestibles

Medicinales

$11.7 \%$

$\square$ Ornamentales

$\square$ Combustible

تConstrucción

шMaderables

¿Forraje

$14.6 \%$

$\square$ Melíferas

SOtros usos

$17.9 \%$

Figura 1. Porcentaje de especies de acuerdo con su utilidad adicional.

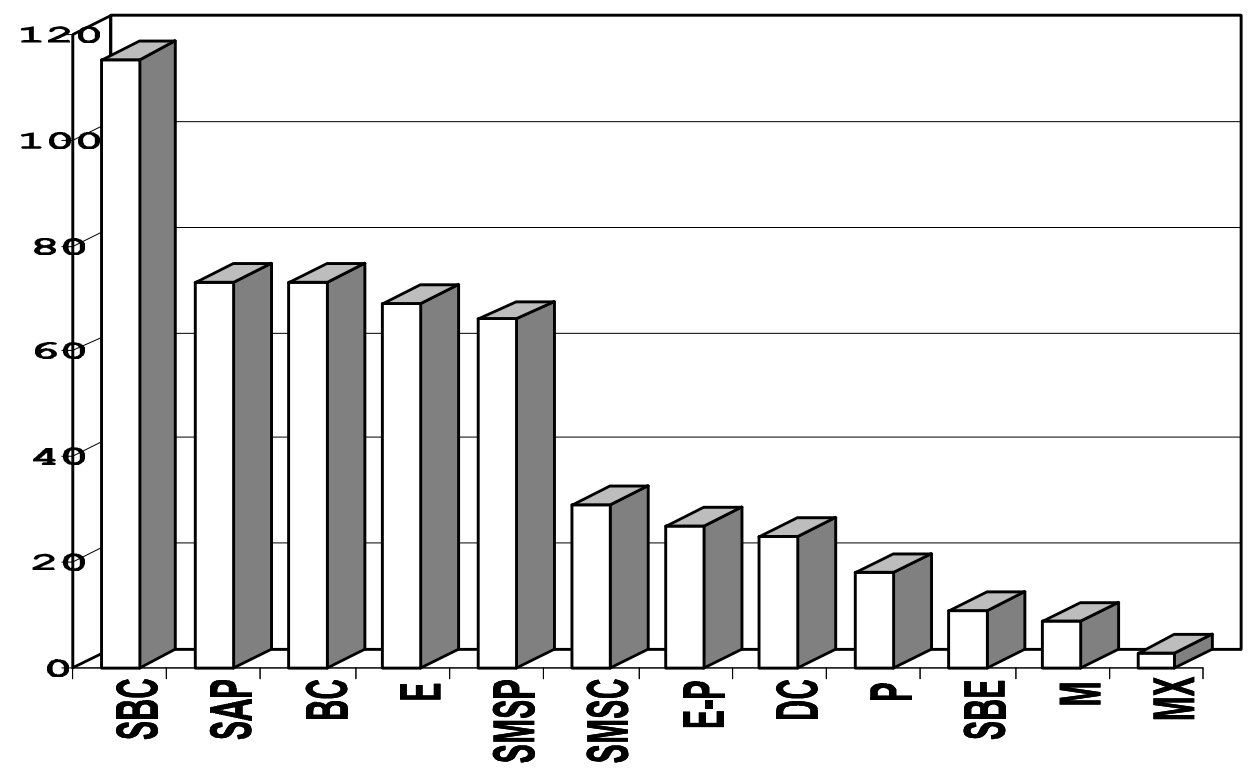

Figura 2. Número de especies por tipos de vegetación.

Abreviaturas:

SBC (selva baja caducifolia)

SAP (selva alta perennifolia)

BC (bosque caducifolio)

$\mathrm{E}$ (encinar)

SMSP (selva mediana subperennifolia)

SMSC (selva mediana subcaducifolia)

E-P (encinar-pinar)

$D C$ (vegetación de dunas costeras)

$P$ (pinar)

SBE (selva baja espinosa)

$M$ (manglar)

MX (matorral xerófilo). 
Guazuma ulmifolia, Jatropha curcas, Leucaena spp., Lippia myriocephala, Melia azederach, Muntingia calabura, Nopalea dejecta, Opuntia strictavar. dillenii, Pachira aquatica, Platycladus orientalis, Prunus serotina subsp. capuli, Pseudobombax ellipticum, Psidium guajava, Randia aculeata, Sambucus nigra, Spondias mombin, Tabebuia chrysantha, T. rosea, Tecoma stans, Thevetia thevetioides, Trema micrantha, Trichilia havanensis y Yucca elephantipes.

\section{DISCUSIÓN}

De acuerdo con los resultados obtenidos, la mayoría de las especies son de uso restringido en determinadas regiones del estado; por otra parte, se observó que los dueños de terrenos que utilizan cercas vivas, han seleccionado las especies preferentemente para satisfacer otras necesidades; así se tiene que las plantas ocupadas para esos fines están vinculadas con las categorías de uso más importantes: alimentaria (hombre, animales domésticos), medicinal, ornamental, en construcción, combustible y melífera; para ello, utilizan tanto especies nativas como algunas introducidas que han demostrado dar mejores resultados.

Otro aspecto importante que vale la pena destacar es la existencia de un gran número de especies faunísticas asociado a esta práctica agroforestal, principalmente aves, reptiles, pequeños mamíferos e insectos. Esto es factible dado que el $25 \%$ de las especies registradas producen frutos y otras estructuras comestibles en alguna época del año.

Por otra parte, para llevar a cabo una selección adecuada de las especies utilizables como cercas vivas, es importante que el campesino, agricultor o propietario del terreno, tenga presente las características que deben reunir las plantas en congruencia con los requerimientos de cercado. También debe ser considerado el período de crecimiento, labores de mantenimiento y productos útiles adicionales que puede obtener de cada una de ellas. Al respecto, se observó en algunos sitios que dada la escasez de especies que convencionalmente se han empleado para este fin, los campesinos han empezado a probar con otras; sin embargo, han manifestado que requieren de una asesoría forestal y apoyo para establecer las especies apropiadas para resolver sus problemas de linderos.

\section{CONCLUSIONES}

El uso de especies nativas como cercas vivas empieza a cobrar mayor importancia principalmente en aquellas áreas donde la vegetación original ha sido perturbada en mayor grado. Es importante destacar el interés que muestra la gente de campo por esta práctica agroforestal, la cual debe ser aprovechada y promovida, si se considera que constituye una de las opciones más viables para la conservación del germoplasma local.

Cabe aclarar que este trabajo constituye un primer intento por dar a conocer las especies que generalmente son empleadas como cercas vivas en Veracruz; por tal motivo, se requiere efectuar estudios detallados que permitan conocer la totalidad de las especies usadas para ese fin en el estado y el manejo que se le brinda a cada una, con el fin de contar con información básica que ayude a proponer su aprovechamiento adecuado, lo cual incidirá en mejores beneficios tanto para los poseedores del recurso como en términos de conservación de los ecosistemas.

\section{RECONOCIMIENTOS}

Al M. en C. Gonzalo Castillo-Campos por las sugerencias hechas al manuscrito, al Biól. Gilberto Cortés Rodríguez por revisar la nomenclatura de los bambúes y al Dr. 
Andrew P. Vovides por la traducción del resumen al inglés.

\section{REFERENCIAS}

Alavez L., S. 1983. Estudio preliminar de los cercos vivos en la ganadería de Teapa, Tabasco; manejo de árboles en los potreros, una técnica silvopastoril. Tesis Ing. Agr. Esp. en Bosques. UACh. Departamento de Bosques. Chapingo, México. 77 p.

Beer J., F. 1994. Consideraciones básicas para el establecimiento de especies maderables en linderos. Agroforestería de las Américas 1: 21 24.

Brummit, R.K. y C.E. Powell (Eds.). 1992. Authors of plant names. Royal Botanic Gardens. Kew. Inglaterra. 732 p.

Castillo-Campos, G. 1991. Vegetación y flora del municipio de Xalapa, Veracruz. Instituto de Ecología, A.C./H. Ayuntamiento de Xalapa/Programa del Hombre y la Biósfera, MAB, UNESCO. Xalapa, Veracruz. $139 \mathrm{p}$.

Chacón E., J.C. 1994. La producción de biomasa en cercos vivos de cocohite Gliricidia sepium (Jacq.) Steud., bajo cuatro frecuencias de poda en Teapa, Tabasco. Séptima Reunión Científica Forestal y Agropecuaria. 6 y 7 de diciembre. Villahermosa, Tabasco. p. 36-38.

Challenger, A. 1998. Utilización y conservación de los ecosistemas terrestres de México. Pasado, presente y futuro. CONABIO, Instituto de Biología, Sierra Madre. México, D.F. 846 p.

Chulim C., R y J. Tun G. 1998. Especies vegetales útiles en las cercas vivas de la Península de Yucatán. Libro de resúmenes del VII Congreso Latinoamericano de Botánica/XIV Congreso Mexicano de Botánica. P. Magaña (ed.). Sociedad Botánica de México. p 146.

García, M.C. 1993. Principales especies forestales tropicales utilizadas en cercos vivos en el estado de Tabasco. Universidad Juárez Autónoma de Tabasco. División Académica de Ciencias Biológicas. Villahermosa, Tabasco, Tab. 81 p.

Instituto de Ecología, A.C. 1994. Flora de Veracruz. Base computarizada de datos florísticos. Xalapa, Veracruz.

López, Y.L. 1990. Cercas vivas en zonas áridas: Agrosilvicultura en el desierto chihuahuense. Tesis Ing. Forestal. División de Ciencias Forestales. UACh. Chapingo, México. 134 p.

Luna M., V.E. 1997. Estudio de vegetación y flora del municipio de Coatepec, Veracruz. Tesis Biól. Facultad de Biología. Universidad Veracruzana. Xalapa, Veracruz. 163 p.

Maldonado M., F., G. Vargas S. y R.F. Molina M. 1998. Los cercos vivos del estado de Tabasco, México. Universidad Juárez Autónoma de Tabasco. División Académica de Ciencias Biológicas. Villahermosa, Tabasco. $71 \mathrm{p}$.

Otárola, A. 1995. Cercas vivas de madero negro: Práctica agroforestal para sitios con estación marcada. Agroforestería de las Américas 2(5):24-30.

Romero, F., C. Chana, J. Montenegro, L.A. Sánchez y G. Guevara. 1991. Productividad de Gliricidia sepium y Erythrina berteroana en cercas vivas manejadas bajo tres frecuencias de poda en la zona atlántica de Costa Rica. Agroforestería No. 6. CATIE. Turrialba, Costa Rica. 
Salinas P., L. y J. Tun G. 1998. Evaluación de la germinación de seis especies dominantes en las cercas vivas. Libro de resúmenes del VII Congreso Latinoamericano de Botánica/XIV
Congreso Mexicano de Botánica. P.Magaña (ed.). Sociedad Botánica de México. p 146.

Zamora C., P. y G. Castillo-Campos. 1998. Vegetación y flora del municipio de Tlalnelhuayocan, Veracruz. Textos Universitarios. Universidad Veracruzana. Xalapa, Veracruz. 82 p.

Anexo 1

Plantas utilizadas como cercas vivas en el estado de Veracruz.

El listado está ordenado alfabéticamente por familia, género y especie. Los nombres de los autores de los nombres científicos se encuentran estandarizados de acuerdo con Brummit y Powell, 1992.

\section{Abreviaturas empleadas. \\ FB (forma biológica) \\ NV (nombres vulgares) \\ TV (tipo de vegetación) \\ OU (otros usos) \\ BC (bosque caducifolio) \\ E (encinar) \\ DC (vegetación de dunas costeras) \\ E-P (encinar-pinar \\ MX (matorral xerófilo) \\ P (pinar) \\ SAP (selva alta perennifolia) \\ SBC (selva baja caducifolia) \\ SBE (selva baja espinosa) \\ SMSC (selva mediana subcaducifolia) \\ SMSP (selva mediana subperennifolia) \\ * (introducida).}

\section{ACANTHACEAE}

Aphelandra deppeana Schltdl. \& Cham.; NV: cola de caballo; FB: arbusto; TV: E, SAP, SBC, SMSP.

Odontonema callistachyum (Schltdl. \& Cham.) Kuntze; NV: canutillo, vara de San Juan; FB: arbusto; TV: BC, SMSP; OU: ornamental; medicinal (anticrotálico y contra erisipela).
ACERACEAE

Acer negundo L. subsp. mexicanum (DC.) Wesm.; NV: abundio, palo blanco; FB: árbol; TV: E-P; OU: ornamental.

\section{ACHATOCARPACEAE}

Achatocarpus nigricans Triana; NV: espino blanco, palo dulce; FB: arbusto; TV: SBC, SMSC; OU: fruto comestible.

\section{AGAVACEAE}

Agave angustifolia Haw.; NV: ixtle, maguey de ixtle, quiote; FB: Herbácea; TV: DC, E, SBC; OU: fibras.

Agave lophanta ; NV: lechuguilla, maguey; FB: arbusto; TV: E, E-P, SBC.

Beschorneria bracteata Jacobi; NV: amole, patamole, plateamole; FB: herbácea; TV: E-P; OU: flor comestible, ornamental. Yucca elephantipes Regel; NV: izote; FB: arbusto; TV: BC, SAP, SBC (cultivada); OU: flor comestible.

Yucca periculosa Baker; NV: Izote; FB: árbol; TV: MX; OU: flor comestible.

\section{ANACARDIACEAE}

Anacardium occidentale L.; NV: marañón; FB: árbol; TV: SBC (cultivada*); OU: fruto comestible.

Comocladia engleriana Loes.; NV: tlatatín, hincha huevos; FB: árbol; TV: SBC, SMSC; OU: fruto comestible.

Mangifera indica L.; NV: mango; FB: árbol; TV: SBC (cultivada*); OU: fruto comestible. 
Schinus molle L.; NV: pirul; FB: árbol; TV: $P, M X$ (cultivada* y naturalizado); OU: corteza en curtiduría, fruto como condimento; medicinal.

Spondias mombin L.; NV: ciruelo agrio, ciruelo de monte, ciruelo silvestre, ciruela de venado, jobo cimarrón; FB; árbol; TV: SASP, SBC; OU: fruto comestible.

Spondias purpurea L.; NV: ciruela, jobo; FB: árbol; TV: SBC; OU: fruto comestible.

\section{ANNONACEAE}

Annona cherimola Miller; NV: chirimoya; anona; FB: árbol; TV: BC, E-P, SMSC; OU: fruto comestible.

Annona muricata L.; NV: guanábana, guanabo; FB: árbol; TV: SBC, SAP; OU: fruto comestible.

Annona purpurea Moc. \& Sessé ex Dunal; NV: ilama, zapote ilama; FB: árbol; TV: SAP, SBC, SMSP; OU: fruto comestible.

Annona squamosa L; NV: anona, chirimoya; FB: árbol; TV: SAP; OU: fruto comestible.

\section{APOCYNACEAE \\ Aspidosperma megalocarpon Muell. Arg.; NV: manzanilla, volador, zapotillo blanco, zapote volador; FB: árbol; TV: SAP; OU: maderable, construcciones rurales, frutos comidos por aves.}

Plumeria rubra L.; NV: cacalosúchil, cacatote, corpus; FB: árbol; TV: DC, SBC, SAP, SMSC; OU: medicinal, flor ceremonial. Stemmadenia donell-smithii (Rose) Woodson; NV: carne de gallina, cojón de burro, cojón de gato, cojón de toro, cundeacán, huevo de burro, huevos de chango, huevo de gato, huevo de mono; FB: árbol; TV: E, SAP, SMSP; OU: látex como pegamento.

Tabernaemontana alba Miller; NV: cojón de gato, cojón de perro, huevos de burro, huevos de gato, lecherillo, yoyo; FB: árbol; TV: DC, SAP, SBC, SMSC; OU: ornamental.

Tabernaemontana citrifolia L.; NV: cojón de gato, huevos de burro, lecherillo, sangrillo; FB: árbol; TV: DC, E, SAP, SMSC; OU: pegamento casero, medicinal. Thevetia ahouai (L.) A. DC.; NV: cojón de venado, huevo de venado, palo de leche, solimán; FB: arbusto; TV: E-P, SAP, SMSP; OU: fruto comestible.

Thevetia thevetioides (H.B. \& K.) Schumann; NV: codo de fraile, solimán; FB: árbol; TV: SBC; OU: ornamental; medicinal; cultivada.

\section{ARALIACEAE \\ Dendropanax arboreus (L.) Decne \&} Planch.; NV: carne de pescado, cucharo, chagane, mano de león, palo de agua, palo cucharo, tamalcohuitl, tamalicahuite; FB: árbol; TV: BC, SAP, SBC, SMSC; OU: cucharas de madera.

Didymopanax morototoni(Aublet) Decne. \& Planch.; NV: marota, primavera; FB: árbol; TV: SAP; OU: muebles finos.

\section{BETULACEAE}

Alnus acuminata Kunth subsp. arguta (Schltdl.) Furlow; NV: aile, ilite; FB: árbol; TV: BC, E-P, P; OU: carbón, leña, construcciones rústicas.

Alnus jorullensis Kunth subsp. jorullensis; NV: ilite; FB: árbol; TV: BC, P; OU: maderable, ornamental, medicinal.

\section{BIGNONIACEAE}

Crescentia alata Kunth; NV: cuatecomate, jícaro; FB: árbol; TV: SBC, SMSP, S; OU: frutos para recipientes, medicinal: oxitócico. Crescentia cujete L.; NV: chomo, jicarera, jicarillo; FB: árbol; TV: BC, SBC, SMSC, P; OU: frutos como recipientes; medicinal: contra la tosferina.

Parmentiera aculeata (Kunth) Seemann; NV: cuajilote, cuachilote, guajilote, chote; FB: árbol; TV: SAP, SBC, SBE; OU: forraje, medicinal (contra la diabetes).

Spathodea campanulata Beauv.; NV: flor de fuego, flor de pato, tulipán africano, tulipán de la India; FB: árbol; TV: BC, E, SBC, SMSP (cultivada*); OU: ornamental. 
Tabebuia rosea (Bertol.) DC.; NV: maculís, primavera, roble, tuxtica; FB: árbol; TV: SAP, SBC, SMSC, Palmar; OU: ornamental, maderable.

Tecoma stans (L.) Juss. ex Kunth; NV: ángel, canario, flor de un día, guachín, San Francisco, sauce, tronador; FB: árbol, arbusto; TV: BC, DC, SBE, SMSC; OU: medicinal: tónico y contra dolores estomacales, ornamental.

\section{BOMBACACEAE}

Ceiba aesculifolia (H.B. \& K.) Britten \& Baker f.; NV: pochote; ceiba, pochote; FB: árbol; TV: SBC; OU: artesanías, relleno de almohadas.

Ceiba pentandra (L.) Gaertn.; NV: ceiba, pochote; FB: árbol; TV: SAP; SMSP; OU: ornamental, relleno de almohadas, medicinal: contra males renales y tratamiento de procesos antiinflamatorios.

Pachira aquatica Aubl.; NV: apompo, chanacol blanco; FB: árbol; TV: SAP, SBC, DC; OU: ornamental; medicinal: contra la diabetes.

Pseudobombax ellipticum (Kunth) Dugand; NV: chanacol, lele; FB: árbol; TV: BC, SAP, SBC, SMSC, SMSP; OU: ornamental, maderable.

\section{BORAGINACEAE}

Cordia alliodora (Ruiz \& Pav.) Oken; NV: hormiguillo, tepesuchi, solerillo, xochicuahuitl; FB: árbol; TV: SAP, SBC; OU: maderable, fruto comestible, medicinal. Cordia dentata Poir.; NV: baboso, gravel, gulaber, moquillo, olavere, zazamil; FB: árbol; TV:, DC, M, SBC, SBE, SAP; OU: maderable, medicinal, fruto comestible.

Cordia megalantha Blake; NV: tres hojas, súchitl, xulaxuchitl; FB: árbol; TV: SAP; OU: construcción.

Ehretia tinifolia L.; NV: borreguillo, frutillo, manzano, manzano coposo; rayado; FB: árbol; TV: BC, E, SBC, SMSP; OU: fruto comestible, hojas medicinales.

Tournefortia glabra L.; NV: cordoncillo, cuerillo, palo de agua, totogapolín, zopilotillo; FB: árbol; TV: BC, E, SAP, SBC,
SMSP; OU: como sombra de café, para amarres.

\section{BROMELIACEAE}

Aechmea bracteata (Sw.) Griseb.; NV: piña, tencolote del grande, tecoloame; FB: hierba; TV: SAP, SBC, SMSP; OU: medicinal: contra hemorragias vaginales; fibra para amarres.

Bromelia pinguin L.; NV: cordón, chiyal; FB: hierba; TV: DC, SBC, SMSP; OU: fruto comestible.

Hechtia podantha Mez.; NV: cordón; FB: hierba; TV: SBC.

\section{BURSERACEAE}

Bursera cinerea Engl.; NV: camarón, camaroncillo, copalillo, mulato, palo mulato; FB: árbol; TV: SBC.

Bursera fagaroides Engl. var. fagaroides; NV: xixote; FB: árbol; TV: MX; OU: leña.

Bursera fagaroides Engl. var. purpusii (Brandegee) McVaugh \& Rzed.; NV: aceitillo, sazafrás; FB: árbol; TV: SBC; OU: medicinal. Bursera graveolens (Kunth) Triana \& Planch.; NV: aceitillo, azafrán, sasafrás, palo de brujo; FB: árbol; TV: SBC; OU: medicinal. Bursera simaruba (L.) Sarg.; NV: chaca; palo mulato, chaca, jiote, mulato, tasun; FB: árbol; TV: DC, E, SAP, SBC, SMSP; OU: medicinal; maderable; frutos alimento para aves.

Protium copal (Schltdl. \& Cham.) Engl.; NV: aceitillo, copal, zapotillo; FB: árbol; TV: SAP; OU: fruto comestible, ceremonias religiosas (resina).

\section{CACTACEAE}

Acanthocereus pentagonus (L.) Britton \& Rose; NV: cruceta; FB: hierba; TV: SBC; OU: comestible.

Nopalea dejecta (Salm-Dyck) Sd.; NV: nopal, chumbera; FB: hierba; TV: DC, E, SBC; OU: comestible, forrajero, medicinal. Opuntia stricta Haw. var. dillenii (Ker Gawler) Benson ; NV: nopal de duna; FB: hierba; TV: DC; OU: comestible.

Stenocereus griseus (Haw.) Buxb. ex Bravo; NV: órgano; FB: arborescente; TV: SBC; OU: frutos comestibles. 


\section{CAPPARIDACEAE}

Crataeva tapia L.; NV: árbol del pepe, chilcoahuitl, pepe, tres hojitas; FB: árbol; TV: DC, SBC, SMSP; OU: fruto comestible.

\section{CAPRIFOLIACEAE}

Sambucus nigra subsp. canadensis; NV: sauco; FB: árbol; TV: BC, E, E-P; OU: fruto como alimento de aves, medicinal, "limpias".

\section{CASUARINACEAE}

Casuarina cunninghamiana Miq.; NV: casuarina, pinito, pino; FB: árbol; TV: BC, SBC (cultivada*); OU: ornamental.

Casuarina equisetifolia L.; NV: casuarina; pino, pino de mar; FB: árbol; TV: BC, DC

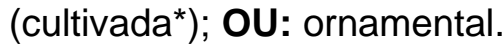

\section{COCHLOSPERMACEAE}

Cochlospermum vitifolium (Willd.) Spreng.; NV: algodón, carne de perro, panicha, palo amarillo, pongalote, pongolote, tecomate; FB: árbol; TV: SBC, SMSP, SAP; OU: ornamental, medicinal, artesanías, relleno de almohadas.

\section{COMBRETACEAE}

Terminalia catappa L. ; NV: almendro; FB: árbol; cultivada; OU: semilla comestible, ornamental, sombra.

\section{COMPOSITAE.}

Tithonia diversifolia (Hemsl.) A. Gray; NV: árnica, árnica de montaña, gigantón, mirasol, tachichi, tamchicht; FB: arbusto; TV: BC, SAP, SBC; OU: contra heridas e hinchazones; melífera.

\section{CONVOLVULACEAE}

Ipomoea carnea Jacq. subsp. fistulosa (Mart. ex Choisy) D.F. Austin; NV: quiebraplato; FB: arbusto; TV: SAP, SMSP, SBC, DC; OU: ornamental.

\section{CUPRESSACEAE}

Cupressus benthamii Endl. var. lindleyi (Klotzsch) Mast.; NV: ciprés; FB: árbol;; TV: BC, E-P, P; OU: ornato, maderable, medicinal, construcciones, cortinas rompevientos.

Juniperus deppeana Steudel var. deppeana; NV: junípero, sabino, ; FB: árbol; TV: E, E-P, P; OU: leña.

Juniperus flaccida Schltdl. var. flaccida; NV: cedro, cedro liso, ciprés, sabino, táxcate; FB: árbol. TV: E, E-P; OU: leña.

Platycladus orientalis (L.) Franco; NV: tuya; FB: arbusto; TV: BC, P-E, P, SBC (cultivada*); OU: ornamental.

\section{EBENACEAE}

Diospyros verae-crucis (Standl.) Standl.; NV: coyolillo, zapotillo; FB: árbol; TV: SBC; OU: fruto comestible, leña.

\section{ELAEOCARPACEAE}

Muntingia calabura L.; NV: puan, capulín, capulín real, hierba de la nigua, mecate de capulín, puyam; FB: árbol; TV: BC, SAP, SBC, SMSP; OU: fruto comestible.

\section{EUPHORBIACEAE}

Croton dioicus Cav.; FB: arbusto; TV: E-P, P.

Croton draco Schltdl.; NV: sangre de grado, sangregado; FB: árbol; TV: BC, SAP, SMSP; OU: medicinal: contra granos bucales.

Croton glabellus L.; NV: agua roja, cascarilla, quina blanca; FB: arbusto; TV: SBC; OU: corteza medicinal, leña.

Euphorbia cotinifolia L.; NV: dólar rojo, mala mujer, mata gallina, trompillo; FB: arbusto; TV: BC; OU: ornamental.

Euphorbia pteroneura A. Berger; FB: arbusto trepador; TV: SBC.

Hevea brasiliensis (Willd.) Müell. Arg.; NV: árbol de hule; FB: árbol; TV: SAP (cultivada*); OU: industrial.

Hura polyandra Baillon; NV: árbol del diablo, árbol del sueño, coatatachi, gavilla, habilla, habillo, jabilla, solimanché; FB: árbol; TV: SAP, SBC; OU: medicinal (purgante y desinfectante), maderable, ornamental. 
Jatropha curcas L.; NV: avellana purgante, chote, piñón, piñón de Indias, piñón purgante, piñoncillo; FB: arbusto; TV: SBC; SMSP; OU: semilla comestible; purgante.

\section{FAGACEAE}

Quercus acutifolia Née; NV: encino duela, encino negro, roble; FB: árbol; TV: E; OU: leña, carbón, medicinal (astringente).

Quercus candicans Née; NV: ixcahuate, chalahuate; FB: árbol; TV: BC, E, E-P; OU: leña, carbón.

Quercus corrugata Hook.; NV: encino, encino blanco; FB: árbol; TV: BC, E, E-P, P; OU: leña y carbón.

Quercus laurina Humb. \& Bonpl.; NV: encino laurelillo, encino memelito, laurelillo; FB: árbol; TV: BC, E, E-P; OU: leña.

Quercus oleoides Schltdl. \& Cham.; NV: encino roble, encino rojo, roble, tesmol; FB: árbol; TV: E, SAP, SBC, SMSP; OU: maderable, leña y carbón.

Quercus peduncularis Née; NV: saucillo; FB: árbol; TV: BC, E, SBC; OU: leña y carbón.

Quercus sapotaefolia Liebm.; NV: encino; FB: árbol; TV: E; OU: duela, carbón.

Quercus xalapensis Humb. \& Bonpl.; NV: encino, encino roble; FB: árbol; TV: BC, E; OU: maderable, leña.

\section{HAMAMELIDACEAE}

Liquidambar macrophylla L. subsp. mexicana Oersted; NV: liquidámbar, ocozotl, ocotzotl, ocoxote, ocozote, techco, copalme; FB: árbol; TV: BC, P, E; OU: herramientas, ornamental.

\section{HERNANDIACEAE}

Gyrocarpus jatrophifolius Domin; NV: volador, carne de perro, palo hediondo; FB: árbol; TV: SBC; OU: frutos empleados como juguetes, se recomienda para elaboración de pulpa de papel.

\section{LAURACEAE}

Litsea glaucescens Kunth; NV: laurel; FB: árbol; TV: BC, E, SBC, SMSP, P; OU: condimento, medicinal.

\section{LEGUMINOSAE.}

Acacia angustissima (Miller) Kuntze; NV: ángel, guajillo, ixtlaxóchitl, jicarillo, mezquite, tepachillo, timbre; FB: árbol; TV: BC, E-P; OU: forraje, licor (raíz); leña; elaboración de canastas.

Acacia farnesiana (L.) Willd.; NV: aroma, espino blanco, espuela de gallo, huichín, huizache, huizache blanco, pedo de burro; FB: arbusto; TV: DC, E, M, SBC, SBE; OU: forraje, medicinal (contra diarrea, la raíz molida para nubes de ojos), maderable, melífera, flores en perfumería, corteza y frutos en curtiduría.

Acacia macracantha Humb. \& Bonpl. ex Willd.; NV: espino, espino blanco; FB: árbol; TV: SBC; OU: postes, leña.

Acacia pennatula (Cham. \& Schlechtendal) Benth; NV: espino, huixtle, concoabite, huizache; FB: arbusto; TV: BC, E, SBC, SMSP; OU: forraje, mangos de herramientas, leña, horcones, postes.

Albizia lebbeck (L.) Benth.; NV: barbas de viejo, chagane, palo de gusano, tijera; FB: árbol; TV: E; OU: ornamental.

Bauhinia divaricata L.; NV: pata de cabra, pata de cochino, pata de vaca, pezuña de venado, xpipe; FB: arbusto; TV: E, SAP, SBC, SMSP; OU: hojas y raíces medicinales; limpias.

Caesalpinia cacalaco Humb. \& Bonpl.; NV: chiquixtle, tehuixtle, tigüixtle, tihuixle, tihuixtle; vaina verde; FB: árbol; TV: SBC; OU: vainas forrajeras, horcones para casas. Caesalpinia mexicana A. Gray; NV: comalillo; FB: arbusto; TV: SBC, SBE; OU: melífera.

Caesalpinia velutina (Britton \& Rose) Standl.; NV: overo, pelguaje; FB: árbol; TV: SBC; OU: maderable, postes.

Cajanus cajan (L.) Millsp.; NV: chícharo, frijol de árbol, kiwistapu; FB: árbol; TV: SMSC; OU: fruto comestible (hervido).

Calliandra houstoniana (Miller) Standl.; NV: acacio, barba de viejo, cabellos de ángel, guajillo, tepeguaje; FB: arbusto; TV: BC, SAP, SBC.

Cassia emarginata L.; NV: frijolillo, palo amarillo, palo hediondo; FB: árbol; TV: 
SBC; OU: maderable, ornamental, leña, horcones.

Cassia fistula L.; NV: caña fístula, flor de mayo, flor de santuario, lluvia de oro; FB: árbol; TV: SBC, SMSC; OU: ornamental.

Delonix regia (Bojer) Raf.; NV: árbol del fuego; framboyán, flamboyán; FB: árbol; TV: SBC (cultivada*); OU: ornamental.

Diphysa robinoides Benth.; NV: amarillo, apipi, chipil, chipitl, ixcuahuite, pionche, pionchi, quebracho, tepezquixtle; FB: árbol; TV: E, DC, SAP, SBC, SMSC; OU: fruto comestible, construcción, leña, ornamental. Enterolobium cyclocarpum (Jacq.) Griseb.; NV: guanacastle, nacaste, nacastle, nacaxtle, orejón, parota; FB: árbol; TV: BC, E, SBC, SMSP; OU: vaina forrajera, construcciones, ebanistería, medicinal; para canoas o cayucos; ornamental.

Erythrina americana Miller; NV: iquimite; colorín, cosquelite, gasparito, pemucha, pichojo; FB: árbol; TV: E; OU: flor comestible, ornamental.

Erythrina berteroana Urban; NV: cosquelite, machetitos, pito; FB: arbusto; TV: SAP.

Erythrina flabelliformis Kearney; NV: tseuse; FB: arbusto; TV: P; OU: medicinal (previene el sarampión).

Erythrina folkersii Krukoff \& Moldenke; NV: colorín, cosquelite, gasparito, iquimite; FB: árbol; TV: BC.

Erythrina herbacea L.; NV: colorín, patol, pichoco de monte; FB: arbusto; TV: E, DC, SBC, SMSP.

Gliricidia sepium (Jacq.) Steudel; NV: cacahuananche, cocohuite; cocuite, cocuitle, cuicuite, mata ratón; FB: árbol; TV: M, SBC; OU: flor comestible, leña, semillas para envenenar ratones.

Inga jinicuil Schltr.; NV: aguatope de montaña, jinicuil; FB: árbol; TV: BC, SMSP; OU: comestible, como sombra de café.

Inga paterno Harms; NV: chalahuite; jinicuil, vainillo; FB: árbol; TV: BC, SAP, SMSP; OU: sombra de cafetales.
Inga vera Willd.; NV: aguatope, chalahuite, guatope de río ; FB: árbol; TV: SBC, SMSP, BC; OU: como sombra de cafetales.

Lennea melanocarpa (Schltr.) Vatke ex Harms.; NV: carga rocío, cocuite, chipiliín, quebruchi, quebracha, quebradiza; FB: árbol; TV: E, SBC, SMSC; OU: forrajera, uso mágico-medicinal.

Leucaena diversifolia (Schld.) Benth.var. diversifolia; NV: guaje, guaje blanco; FB: árbol; TV: BC, SBC, SMSP; OU: semilla comestible, leña, sombra.

Leucaena lanceolataS. Watson; NV: guaje de indio, guaje de monte; FB: árbol; TV: SBC, SMSC; OU: semillas comestibles, melífera.

Leucaena leucocephala (Lam.) De Wit subsp. glabrata (Rose) Zárate; NV: guaje; FB: árbol; TV: E, SBC, SMSP.

Leucaena leucocephala (Lam.) De Wit subsp. leucocephala; guaje; FB: árbol; TV: SBC, SBE, SMSP; OU: semillas comestibles, forraje, leña.

Leucaena pulverulenta (Schltr.) Benth. subsp. pulverulenta; NV: guaje rojo; FB: árbol; TV: BC, SAP, SMSP; OU: semilla comestible, flores melíferas, como sombra.

Lonchocarpus guatemalensis Benth.; NV: cimatl, frijolillo, gusano, marinero de montaña, palo de suelo, palo de tepache, palo gusano, rosa morada; FB: árbol; TV: E, SAP, SMSP; OU: como sombra.

Lysiloma acapulcensis (Kunth) Benth; NV: guaje sabana, guayal, soncabite hembra, tepeguaje; FB: árbol; TV: E, SBC, SAP; OU: postes.

Lysiloma divaricata (Jacq.) Macbr.; NV: guajillo, tepeguaje; FB: árbol; TV: SBC.

Parkinsonia aculeata L.; NV: cuajilote, chote, guachilote, pepino silvestre, retama; FB: árbol; TV: SBC, SBE; OU: medicinal.

Piscidia piscipula (L.) Sarg.; NV: chijol, habín, jabín; FB: árbol; TV: E, SAP, SBC; OU: construcciones rurales.

Pithecellobium arboreum (L. Urban); NV: cañamazo, camaronera, coralillo, frijolillo, papalote, rabo de lagarto, zancuabite, zoncoabite; FB: árbol; TV: BC, E, SAP, SMSP; OU: fustes, como sombra. 
Pithecellobium calostachys Standl.; NV: ahogador, amuchile, guamúchil, ogador, palo de humo; FB: árbol; TV: SBC, SMSP; OU: vainas comestibles; cabos de herramientas.

Pithecellobium dulce (Roxb.) Benth.; NV: guambuche, guamúchil, muchite; FB: árbol; TV: SBC; OU: arilo comestible, melífera.

Pithecellobium lanceolatum (Humb. \& Bonpl. ex Willd.) Benth.; NV: guamuchete, jinicuilillo, guamúchil, muchite, peleple; FB: árbol; TV: DC, SBC, SBE, M, SMSC; OU: arilo de la semilla comestible.

Pithecellobium mangense (Jacq.) Mcbride; NV: moreno; FB: arbusto; TV: SBC; OU: construcción rural; forraje, leña, postes.

Tamarindus indica L.; NV: tamarindo; FB: árbol; TV: E, SBC, SMSC (cultivada*); OU: fruto comestible, sombra, ornamental, forraje de ganado, medicinal: laxante.

\section{LOGANIACEAE}

Buddleia americana L.; NV: pozancle, sompantle, tepozán; FB: arbusto, árbol; TV: BC, SBC, SBE; OU: medicinal: antiinflamatorio, contra reumas.

Buddleia cordata Kunth subsp. cordata; NV: sallolisca, somantle, tepozán, tepozán blanco, xompantle; FB: arbusto, árbol; TV: $\mathrm{BC}, \mathrm{E}$; OU: forrajero; diurético.

\section{LYTHRACEAE}

Adenaria floribunda H.B. \& K.; FB: arbusto; TV: DC.

Ginoria nudiflora (Hemsl.) Koehne; NV: agame, guayabillo, pimientillo; FB: arbusto; TV: SBC.

\section{MAGNOLIACEAE}

Talauma mexicana (DC.) Don; NV: flor de atole, flor de corazón, magnolia, maynacoi, súchil, yolo, yoloxóchitl; FB: árbol; TV: BC, SAP, SMSP; OU: bebida (pétalos); medicinal (enfermedades cardíacas).

\section{MALPIGHIACEAE}

Byrsonima crassifolia (L.) Kunth in H.B.K.; NV: nance, nanche, nanchi, nantzincuahuitl;
FB: árbol; TV: E, SAP, SBC; OU: fruto comestible; elaboración de licores; corteza y hojas medicinales.

\section{MALVACEAE}

Hibiscus rosa-sinensis L.; NV: gallarde, lamparilla, obelisco, rehilete, rosa china, súchel, tulipán, tulipán moteado, tulipán pinto, tulipán relleno; FB: arbusto; TV: BC, SBC, SMSP (cultivada*); OU: ornamental.

Malvaviscus arboreus Cav.; NV: gapache, ixhuaquelt, majagüilla, manzanita, manzanillo, monacillo rojo; FB: arbusto; TV: BC, SMSP; OU: ornamental, medicinal (hojas como antifebrífugo).

\section{MELIACEAE}

Cedrela odorata L.; NV: cedro rojo; FB: árbol; TV: SAP, SBC, SMSP; OU: muebles finos, construcción.

Melia azedarach L.; NV: cresmo, maravilla, paraíso, piocha, pionche, primavera; FB: árbol; TV: BC, E, SMSP; OU: ornamental, medicinal (contra dolor de estómago), maderable.

Trichilia havanensis Jacq.; NV: cucharo, estribillo, garrapatillo, lomo prieto, palo cucharo, rama tinaja; FB: árbol; TV: BC, E, SMSP; OU: ornamental.

Trichilia hirta L.; NV: cedrillo, ciruelillo, ciruelillo cimarrón, tepexoco; FB: árbol; TV: SAP, SBC, SMSC.

\section{MORACEAE}

Castilla elastica Sessé; NV: árbol del hule, árbol del pato de hule cimarrón, hule, julián, kut; FB: árbol; TV: SAP, SMSC cultivada*); OU: goma, mangas de hule, ornamental. Chlorophora tinctoria (L.) Gaudich.; NV: árbol de mora, moral; FB: árbol; TV: $\mathrm{M}$, SBC, SMSC; OU: fruto comestible, sombra de café, maderable.

Ficus cotinifolia Kunth; NV: higuera, planta de hule; FB: árbol; TV: SBC, SAP, SMSC.

Trophis mexicana (Liebm.) Bur.; NV: chichilillo, gusano, lecherillo de monte, palo gusano, palo verde, paque, ramoncillo; FB: árbol; TV: BC, E, SAP. 
Trophis racemosa (L.) Urban; NV: chichimón, lecherillo de montaña, palo de chichimón, palo verde; ramoncillo, tepozontla, zacate ramón; FB: árbol; TV: BC, E, M, SAP, SBC, SMSP.

\section{MYRTACEAE}

Eugenia capuli (Cham. \& Schltdl.) O. Berg; NV: capulín, capulín de mayo, capulín de zorrillo, capulincillo, clavo, clavero, chepecuy, escobillo, escobilla, quinda, palo de temazate, raigán, romerillo, tecapule, viscolín; FB: árbol; TV: BC, E, SMSP; OU: fruto comestible, cabos de azadón.

Psidium guajava L.; NV: guayaba; FB: árbol; cultivada; TV: BC, E, SBC, SAP, SMSP; OU: fruto comestible, medicinal.

Syzygium jambos (L.) Alston; NV: laurel, lele, poma, poma rosa; FB: árbol; TV: BC, E, SBC, SMSP; OU: fruto comestible, leña.

\section{NYCTAGINACEAE}

Bouganvillea glabra Choisy; NV: bugambilia; FB: árbol; TV: SBC, SMSC

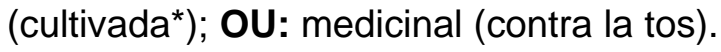

\section{OLEACEAE}

Fraxinus schiedeana Schltdl. \& Cham.; NV: escobo, fresno; FB: árbol; TV: SBC, SMSC; OU: maderable, construcciones rurales.

\section{OPILIACEAE}

Agonandra obtusifolia Standl.; NV: limoncillo, tepechote amarillo, nopalillo; FB: árbol; TV: SBC, SBE.

\section{PALMAE .}

Acrocomia mexicana Karw. ex Mart.; NV: coyol, coyol baboso, coyol redondo, palma; FB: árbol; TV: SAP, SBC, SMSC; OU: fruto comestible.

Chamaedorea tepejilote Liebm. ex Mart.; NV: tepejilote; FB: arbusto; TV: SAP; SMSP; OU: ornamental; inflorescencia masculina comestible.

Cocos nucifera L.; NV: coco; FB: árbol; TV: DC, SBC (cultivada*); OU: fruto comestible, artesanías.
Sabal mexicana Mart.; apachite, palma apachite, palma redonda, guano; FB: árbol; TV: E, P, SBC, DC; OU: artesanías, techados, frutos comestibles.

Scheelea liebmannii Becc.; NV: coyol real, palma real; FB: árbol; TV: SMSC; OU: fruto comestible, construcciones rurales.

\section{PAPAVERACEAE}

Bocconia frutescens L.; NV: barbasco, llora sangre, gordolobo, hoja ceniza; FB: arbusto; TV: BC, E, E-P, SAP, SBC; OU: medicinal, infecciones de la piel.

\section{PINACEAE}

Pinus ayacahuite Ehrenb. ex Schtdl.; NV: acalocote, ayacahuite; FB: árbol; TV: E-P; OU: maderable, tejamanil para techos de casas, leña.

Pinus montezumae Lamb.; NV: pino blanco, chamaite blanco; FB: árbol; TV: P; OU: leña.

Pinus oocarpa Schiede ex Schltdl.; NV: pino; FB: árbol; TV: P; OU: leña.

Pinus patula Schltdl. \& Cham.; NV: pino colorado, pino rojo, ocote rojo; FB: árbol; TV: E-P; OU: leña.

Pinus pseudostrobus Lindl. var. apulcensis (Lindley) Stead; NV: pino; FB: árbol; TV: E-P; OU: leña.

Pinus pseudostrobus Lindl. var. pseudostrobus; NV: pino real; FB: árbol; TV: P; OU: leña.

\section{POACEAE}

Arundo donax L.; NV: tarro, carrizo rayado; FB: arbustiva; TV: BC, DC, E, SBC; OU: ornamental; artesanías, construcciones rurales.

Bambusa vulgaris Schrad. ex J.C. Wendl.; NV: bambú, bambú rayado, caña de otate, otate; FB: arbustiva; TV: BC, E; OU: ornamental, techos de casas, escobas, escobillas.

Guadua aculeta (Rupr.) A.S. Hitchc.; NV: bambú, carrizo, matlok, otate, tarro; FB: arbustiva; TV: E, E-P, OU: construcciones rurales. 
Guadua amplexifolia (Fourn.) McClure; NV: bambú, caña vaquera, otate, tarro; FB: arbusto; TV: SAP, SBC, SMSP, P; OU: construcciones rurales.

Guadua Iongifolia (Fourn.) McClure; NV: caña de otate, cañizo, jimba, jimba, otate; FB: arbustiva; TV: SAP; OU: medicinal: contra afecciones renales.

Guadua vulgaris Schrader ex Wendl; NV: bambú, bambú rayado, caña de otate, otate; FB: arbustiva; TV: BC, E; OU: ornamental, techos de casas, escobas, escobillas.

Otatea recta Soderstrom; NV: jimba; FB: arbustiva; TV: SAP; OU: construcción.

\section{POLYGONACEAE}

Coccoloba barbadensis Jacq.; NV: guerro, hojancho, uvero; FB: arbusto; TV: DC, SBC, SAP, SMSP; OU: construcciones rurales, medicinal.

Coccoloba uvifera L.; NV: uvero, uvero de playa; FB: arbusto; TV: DC; OU: frutos comestibles.

\section{RHAMNACEAE}

Ziziphus amole (Sessé \& Moc.) M.C. Johnst.; NV: brasil; FB: árbol; TV: SBC; OU: frutos comestibles, postes.

\section{RHIZOPHORACEAE}

Rhizophora mangle L; NV: mangle rojo, mangle colorado; FB: árbol; TV: manglar; OU: leña, carbón, construcciones rurales, curtiduría y tinción.

\section{ROSACEAE}

Crataegus mexicana Mociño \& Sessé; NV: tejocote agrio, tejocote silvestre; FB: árbol; TV: BC, E-P, P; OU: frutos comestibles; construcción.

Crataegus pubescens; NV: cojote, tejocote; FB: árbol; TV: E-P, BC; OU: comestible, construcción.

Eriobotrya japonica (Thunb.) Lindl.; NV: níspero; FB: árbol; TV: $\mathrm{BC}, \mathrm{E}, \mathrm{SMSP}$ (cultivada); OU: fruto comestible, ornamental .
Prunus serotina Ehrenb. subsp. capuli (Cav.) McVaugh; NV: capulín; FB: árbol; TV: BC, E, E-P; OU: frutos comestibles.

\section{RUBIACEAE}

Genipa americana L.; NV: jagua, yagua, yoale, yual, yuale; FB: árbol; TV: E, SAP, SMSP; OU: fruto comestible.

Randia aculeata L.; NV: crucero, cruceto, crucetillo, crucetilla blanca, torillo, torito, xilimayatl; FB: árbol; TV: DC, E, SAP, SBC, SMSP.

Randia xalapensis M. Martens \& Galeotti; NV: cruceta; FB: arbusto; TV: BC, E.

\section{RUTACEAE}

Citrus aurantium L.; NV: naranjo, naranjo agrio; FB: árbol; cultivada*; OU: fruto comestible, medicinal.

Citrus sinensis (L.) Osbeck.; NV: naranjo, naranjo dulce; FB: árbol, cultivada*; OU: fruto comestible, medicinal.

Esenbeckia berlandieri Baillon subsp. berlandieri; NV: hueso de tigre, paguay, pahuash; FB: árbol; TV: SBC, SBE, SMSC. Zanthoxylum fagara (L.) Sarg.; NV: naranjillo, uña de gato, zarza de árbol; FB: árbol; TV: BC, DC, E, SBC; OU: medicinal (tosferina).

\section{SALICACEAE}

Populus mexicana Wesmael; NV: álamo; FB: árbol; TV: BC, SMSP; OU: maderable, ornamental.

Salix humboldtiana Willd; NV: sabino, sauce; FB: árbol; TV: E, SBC, SMSP; OU: cestería, muebles, corteza medicinal, postes.

\section{SAPINDACEAE}

Cupania dentata DC.; NV: cañilla de venado, cosalcahuite, espraguello, guacamayo, quiebra cuchillo, quiebra hacha, tepeshi, tepexín, tres lomos, zapotillo, zapotito; FB: árbol; TV: BC, SAP, SMSP; OU: maderable, leña. 
Cupania glabra Sw.; NV: cojote venado, cosalcahuite, palo de piedra, quiebra hacha, quebracho colorado, tepeshi, tepesi, tres Iomos; FB: árbol; TV: SAP.

Dodonaea viscosa (L.) Jacq.; NV: camarón, chapulistli, duraznillo, ocotillo; FB: arbusto; TV: E, E-P, SBC, SMSC; OU: medicinal.

Sapindus saponaria L.; NV: chololo, chololote, gualul, jaboncillo, zapotillo; FB: árbol; TV: BC, SBC, SMSP; OU: frutos usados como detergente de ropa.

\section{SAPOTACEAE}

Bumelia celastrina Kunth; NV: pionche; FB: arbusto, árbol; TV: SBC, SMSC; OU: frutos comestibles, postes, leña.

Chrysophyllum cainito L.; NV: caimito; FB: arbusto, árbol; TV: SAP, SBC; OU: fruto comestible.

Dipholis salicifolia (L.) A. DC.; NV: capulín, zapotillo; FB: arbusto, árbol; TV: E, SBC.

Pouteria sapota (Jacq.) H.E. Moore \& Stearn; NV: mamey, zapote, zapote mamey; FB: árbol; TV: SAP, SMSP; OU: frutos comestibles.

\section{SIMAROUBACEAE}

Simarouba glauca DC.; NV: aceituno, palo gusano; FB: árbol; TV: E, SAP, SMSP.

\section{SOLANACEAE}

Brugmansia suaveolens (Willd.) Bercht. \& J. Presl.; NV: floribundio, floripondio, toloache; FB: arbusto; TV: BC, E, E-P, SMSP (cultivada*); OU: ornamental.

Brugmansia candida Pers.; NV: floripondio; FB: arbusto; TV: BC; E-P, SMSP (cultivada*); OU: ornamental, medicinal: desinflamante.

Cestrum dumetorum Schltdl.; NV: chacuaco, galán, huele de dia, huele de noche, teozán, horcajudas; FB: arbusto; TV: DC, SBC, SMSP; OU: esencia.

Cestrum elegans (Brongn.) Schltdl.; NV: aretillo, hierba del espanto; FB: arbusto; TV: BC, E, SAP, SMSP; OU: medicinal: para curar el "espanto", ornamental.
Cestrum glanduliferum Francey; NV: huele de noche; FB: arbusto; TV: SAP, SMSP.

Cestrum nocturnum $L$.; NV: huele de noche; FB: arbusto; TV: BC, E, SMSP; OU: ornamental.

Solanum jasminoides Paxton; NV: enredadera blanca, gloria, hiedra; FB: trepadora (cultivada*); OU: ornamental.

Solanum wendlandii Hook. f.; NV: hiedra; FB: árbol; TV: BC, SAP, SBC; OU: ornamental

\section{STERCULIACEAE}

Guazuma ulmifolia Lambert; NV: cuázima, cuaulote, guázima, guázimo, guázimo cuaulote, guázumo, hierba del tapón, tapa culo; FB: árbol; TV: M, SAP, SBC, SMSC; OU: frutos comestibles, maderable, medicinal (corteza como antifebrífugo, contra la diarrea), postes, leña, forraje (hojas).

\section{THYMELACEAE}

Daphnopsis americana (Mill.) J.R. Johnst.; NV: jonote, nopo, palo de nopo, pescador; FB: arbusto; TV: E, SBC, SMSP.

\section{TILIACEAE}

Heliocarpus mexicanus (Turcz.) Sprague; NV: jonote; FB: arbusto, árbol; TV: E; OU: corteza para papel.

\section{ULMACEAE}

Trema micrantha (L.) Blume; NV: capulín, mata caballo, palo de ishpepe, ixpepe; FB: árbol; TV: SBC, SMSP, E, BC, E-P; OU: construcciones rurales, papel, medicinal (contra sarampión).

Ulmus mexicana (Liebm.) Planchon; NV: cempoalébatl, cuero, cuerillo, olmo; FB: árbol; TV: BC, SAP, SMSP; OU: leña, carbón, maderable, construcciones rurales.

\section{URTICACEAE}

Myriocarpa longipes Liebm.; NV: apante, cocujala, chalagogue, chichicastlillo, chifla culo, chiguapo, palo de agua, palo de fideo, totonaxclillo; FB: árbol; TV: BC, SAP, SMSP. 
VERBENACEAE

Citharexylum ellipticum D. Don; NV: capulincillo, nacahuite; FB: arbusto; TV: DC, M, SAP; OU: comestible, medicinal.
Duranta repens L.; NV: corona de novia, espino blanco, garbancillo; FB: arbusto; TV: BC, E, P, SMSP; OU: ornamental.

Lippia myriocephala Schltdl. \& Cham.; NV: colpanchi, colpanchi blanco, palo de gusano; FB: árbol; TV: BC, E, M, P, SAP; OU: construcciones rurales, leña. 